\title{
Sampling and multiplexing in lab-on-paper bioelectroanalytical devices for glucose determination
}

\author{
O. Amor-Gutiérrez, E. Costa-Rama, M.T. Fernández-Abedul* \\ Departamento de Química Física y Analítica, Facultad de Química, Universidad de \\ Oviedo, 33006 Oviedo, Spain \\ *e-mail: mtfernandeza@uniovi.es \\ *Tel.: +34985102968
}

\section{Abstract}

In this work, we present a multiplexed (eight simultaneous measurements) paperbased electrochemical device developed in a very simple way and using low-cost materials, such as paper, carbon ink and multifunctional connector headers. Meanwhile, we have also combined the paper-based electrochemical platform with a glass-fiber strip in order to integrate easily a sampling step. Both approaches, simultaneous measuring and sampling, have been applied to the determination of glucose using bienzymatic biosensors. They are fabricated by adsorbing the mixture of enzymes (glucose oxidase and horseradish peroxidase), as well as the ferrocyanide, mediator of the electron transfer, on the paper-based electrode. After drying, the measuring solution (containing either glucose standards or samples) is added and the eight corresponding chronoamperograms are recorded. In the case of the microfluidic approach for sampling purposes, the glass-fiber pad (sampler) is immersed in a container with the solution, which flows by capillarity until it reaches the working electrode. The integration of one more step of the analytical process advances towards real and useful lab-on-a-chip devices. With these designs, a linear range comprised between 0.5 and $15 \mathrm{mM}$ was achieved for glucose determination, with excellent precision. If the sampler is employed, it is not necessary to use micropipettes and, nevertheless, precise measurements are obtained. The RSD of the slopes obtained for different calibrations performed in different days, with different arrays of electrochemical cells and different solutions is ca. $1 \%$. Accurate results are obtained in the determination of glucose in real samples (orange fruit and cola beverages).

\section{Keywords}


Paper-based electroanalysis, lab-on-paper, sampling, multiplexing, paper-based biosensor, bioelectroanalytical platforms, enzymatic analysis, glucose, low-cost analysis.

\section{Introduction}

The introduction of microfluidic paper-based analytical devices ( $\mu$ PADs) by Whitesides and co-workers in 2007 (Martinez et al., 2007) has opened a new and huge field of research. Although paper has been employed for analytical purposes for many years, this material has been revisited for different and interesting applications (Cate et al., 2015; Cunningham et al., 2016; Mettakoonpitak et al., 2016; Yang et al., 2017). When compared with conventional microfluidic analytical devices, usually using silicon, glass or polymers as substrates, $\mu$ PADs are simple, user-friendly, miniaturized, ubiquitous, affordable and disposable (Martinez et al., 2010). Regarding detection, electrochemical sensing is ideally suited for $\mu$ PADs due to the availability of portable potentiostats for on-site measurements (Ainla et al., 2018; Nemiroski et al., 2014), the low operating costs and easy handling and the possibility of easily fabricating miniaturized electrodes onto paper (Hamedi et al., 2016; Mettakoonpitak et al., 2016). Compared to colorimetric techniques, which are most commonly used with PADs (Yang et al., 2017), electrochemical detection generally exhibits lower response times and higher sensitivities, and, in addition, it is not affected by the color of the sample.

For these reasons, integrated systems that combine paper-based microfluidic components and electrochemical detection have a great innovating potential to generate devices for their use in simple, rapid and decentralized analysis, as demonstrated by the wide range of different electrochemical paper devices reported to date (Arduini et al., 2017; Hamedi et al., 2016; Mettakoonpitak et al., 2016; Nie et al., 2010). On the other hand, the lab-on-a-chip concept can also be applied to these paper-based devices (lab-on-paper), with the integration of different operations of the analytical process in simple and miniaturized devices (Cinti et al., 2017b; Nguyen et al., 2018; Xu et al., 2018; Yager et al., 2006). In this way, bioassays, either enzymatic (Zhao et al., 2013), immunological (MazzuNascimento et al., 2017) or DNA-based (Song et al., 2018; Teengam et al., 2017) have taken advantage of many properties of paper, such as its ease of storage, porosity or foldability. 
One of the most attractive features of these "pocket-size" platforms is their simplicity and ease of fabrication. The most widespread methods for the fabrication and integration of electrodes on paper-based platforms consist in the deposition of conductive materials by drawing (Russo et al., 2011) or printing (Tobjörk and Österbacka, 2011; Yamada et al., 2015), mostly depending on the state of the material. A pencil can incorporate solid composite materials that can be transferred to a piece of paper (Bernalte et al., 2016; Dossi et al., 2014). In this way, carbon electrodes can be fabricated with or without employing stencils, using pencils or pens directly, following a pattern previously designed. On the other hand, ink dispersions can also be deposited and cured on metallic mass-produced elements (Nanni et al., 2018; Rama et al., 2017) that can be further integrated on adequate platforms.

Screens or stencils with optimized designs can be used to print ink dispersions on flat surfaces by hand or by employing automatic screen-printing machines that contain conductive components. Then, solvent evaporation by curing can produce complete electrochemical cells on paper (Cinti et al., 2017a). Ink can be also loaded in a ball-pen actuated using a computer-controlled plotter in order to obtain higher precision (Glavan et al., 2014) or in ink cartridges that operate with office printers (Yamada et al., 2015). In all cases, the geometry of the electrodes in the electrochemical cell needs to be carefully optimized to achieve adequate analytical features. Mostly, all the three electrodes of a potentiostatic electrochemical cell (working, reference and auxiliary electrodes) are printed. The pattern (designed using a software or transferred from a stencil/screen) is required to avoid contact between electrodes and to define the geometry. This implies an increase in cost and fabrication time. We have previously overcome these limitations by combining a paper-based carbon working electrode (WE) with external reference (RE) and auxiliary electrodes (AE). Thus, not only stencil printing is avoided, but also the procedure is simplified, and the cost reduced. In stencil- or screen-printing procedures some ink is wasted, but since a pattern here is not required, ink can be easily deposited with a micropipette. External electrodes are placed using racks of gold-plated pins which can be found in the market as standard electronic connections (Amor-Gutiérrez et al., 2017). These racks also work as interface between the three electrodes and the potentiostat, by using a commercial connector. Moreover, although gold pins can be considered disposable due to their low cost, in this case they can be reused. Then, the paper-based WEs can be easily changed between measurements as in the case of commercial screen- 
printed electrodes (SPEs). Once we demonstrated that this electrochemical cell, which combines paper-based and wire electrodes, works adequately as a biosensor transducer (Amor-Gutiérrez et al., 2017), the next steps that we depict here are: i) to develop a multiplexed device to perform simultaneous measurements, taking advantage of the connectors available in the market and ii) to integrate sampling, one more functionality besides enzymatic reaction and detection, approaching the lab-on-paper concept.

In this work, we describe the development of an 8-channel platform where all the electrochemical cells are constructed in the same "card", combining eight different paper-based WE with a commercial electronic connection that provides eight AEs and eight REs, as well as the interface with the potentiostat. The construction of eight electrochemical cells has been chosen due to the availability of 8-channel micropipettes as well as multichannel connectors and multipotentiostats, which are commercially available. However, the simple design of the platform allows to obtain "cards" with more electrochemical cells. It is important to note that this multiplexed platform fulfils important requirements of Green Analytical Chemistry (Gałuszka et al., 2013), since it allows to perform multiplex on-site measurements using a miniaturized device, minimizing the volume of reagents required and the amount of waste produced (the connector is reused and only the paper-WE is discarded). In the scientific literature there are many different examples of multiplexed electrochemical systems for biological applications (Liao et al., 2018; Martín-Yerga et al., 2013; Zhao et al., 2013) but, to the best of our knowledge, this is the first time that a multiplexed electrochemical platform is combined with low-cost goldplated connector headers to perform simultaneous measurements. This could also be an interesting approach to detect different analytes in a single card, since each electrochemical cell of the platform (also referred to as node) can work individually. Then, this multiplexed platform can be used to detect several analytes in one sample just by varying the sensor phase in different electrodes of the card.

The second challenge focuses on another important principle of Green Chemistry: the automation of methods (Gałuszka et al., 2013). Taking this into account, we propose the use of a microfluidic "tongue" which allows automatic sample collection, transporting it from the sample container to the electrochemical cell. The inclusion of this step, performed in a precise way, allows to advance in the concept of lab-on-a-chip devices, generating real lab-on-paper analytical platforms. This sampling "tongue" is made of glass fiber pads, commonly used in lateral flow 
immunoassays (Rodríguez et al., 2016), but this is the first time it is used in the development of electrochemical biosensors. The feasibility of using the multiplexed platform for the development of biosensors was tested applying it to the construction of a glucose enzymatic sensor. Glucose is one of the most important biological compounds for life and there are many analytical methods to quantify it, as it is closely related to human diabetes (Galant et al., 2015). However, the development of low-cost yet precise methodologies is still a challenge.

\section{Materials and Methods}

\subsection{Chemicals}

Glucose oxidase (GOx), horseradish peroxidase (HRP), potassium ferrocyanide, Trizma ${ }^{\circledR}$ base, methylene blue and the Glucose Assay Kit were provided by Sigma-Aldrich. Anhydrous $D-(+)$-glucose was purchased from Merck. Carbon ink was provided by Gwent Electronic Materials Ltd. Ultrapure water obtained from a Millipore Milli-Q purification system (Millipore Direct- $Q^{\mathrm{TM}} 5$ ) was used throughout this work. All chemicals were of analytical reagent grade.

Glucose stock solutions were prepared daily in a $0.1 \mathrm{M}$ Tris- $-\mathrm{HNO}_{3} \mathrm{pH} 7.0$ buffer solution. This buffer and the solutions of GOx and HRP were prepared weekly (in Tris- $\mathrm{HNO}_{3}$ buffer solution) and stored at $4^{\circ} \mathrm{C}$. Potassium ferrocyanide was prepared daily in the Tris- $\mathrm{HNO}_{3}$ buffer solution. The $\mathrm{pH}$ value for the buffer solution has been previously optimized in our research group, when developing the first enzymatic glucose biosensor using screen-printed carbon electrodes (Biscay et al., 2011).

\subsection{Apparatus and Measurements}

Measurements were performed using a $\mu$ Stat 8000 potentiostat (DropSens) interfaced to a computer system controlled by DropView 84002.0 software. All measurements were carried out at room temperature. A multichannel connector (ref. DRP-CAST1X8) purchased from DropSens was used as interface between the multisensor platform and the potentiostat to perform the measurements. Whatman $^{\mathrm{TM}}$ paper $(100 \times 300 \mathrm{~mm})$ and a wax printer XEROX ColorQube 8570 were used for the fabrication of paper-based electrodes. Gold-plated connector headers were delivered by Digikey. Glass-fiber sample pads were purchased from Merck Millipore. 


\subsection{Electrochemical cells}

All the studies were performed using paper-based working electrodes and the goldplated pins of commercial connector headers as reference and auxiliary electrodes. One gold pin is also used as connection of the working electrode to the potentiostat. The procedure for printing the pattern of the hydrophobic area, diffusing the wax and depositing the carbon ink was previously described for single platforms by our research group (Amor-Gutiérrez et al., 2017). In this work, we have used pieces of paper containing eight (Figure S1) or one (Figure 2) patterned circles. Then, the paper layer containing carbon ink (paper-based WEs with a diameter of $4 \mathrm{~mm}$, i.e., an area of $12.6 \mathrm{~mm}^{2}$ ) is placed between the pins of the connector header, one at the bottom and two at the top. Paper is always cut to warranty that the distance between the paper-based working electrodes and the gold-plated connector is always the same. For each electrochemical cell (from now referred to as node), the pin at the bottom connects with the carbon ink of the working electrode. The other two, on the opposite side, act as reference and auxiliary electrodes (Figure 1a). Once the paper is clipped, it is inserted in a commercial interface to connect to the potentiostat (Figure 1b).

Then, the solution is added on the opposite side of the ink (top side), contacting with auxiliary and reference electrodes as well as with the ink (through the paper by capillarity). In this way there is ionic contact between the three electrodes, closing the circuit when the potential is applied, and producing the flow of current. Chronoamperograms like those in Figure 1c are obtained simultaneously.

While the paper-based WEs are disposable, the gold-plated connector can be either disposed or reused. Along this work we have reused it without experimenting problems of cross-contamination, washing it with Milli-Q water before each use.

\subsection{Sampler integration}

The microfluidic "tongue" or sampler was made of a glass-fiber conjugate pad. After being cropped to be T-shaped, it was placed between the paper-based electrode and reference and auxiliary electrodes. Then, there is no need to use adhesives or any kind of fixing. The microfluidic tongue fits perfectly with the WE, as the upper part of the $T$ has the same width as the diameter of the WE. To perform the sampling, the tongue is brought into contact with the solution, letting the liquid rise by capillarity until it wets completely the circular working area. By this methodology of assembling all the materials (Figure 2a), a multilayer device is 
obtained, as seen in Figure $2 \mathrm{~b}$ and the schematics of Figure S2. As in the case of the array, the multilayer paper-platform is single-use and the connector can be reused after a washing step with Milli-Q water.

\subsection{Electrode (bio)modification}

The concentration of GOx and HRP enzymes, as well as this of the mediator, ferrocyanide, were previously optimized in our research group (Amor-Gutiérrez et al., 2017). Thus, a cocktail of enzymes and mediator, with $1.6 \mathrm{U} / \mu \mathrm{L}$ of GOx, 2.5 $\mathrm{U} / \mu \mathrm{L}$ of $\mathrm{HRP}$ and $0.1 \mathrm{M}$ of ferrocyanide, was prepared in $0.1 \mathrm{M}$ Tris $-\mathrm{HNO}_{3}$ buffer solution $\mathrm{pH} 7.0$ and deposited on the paper-based working electrode (Figure 1d), at the opposite side of the carbon ink. A volume of $5 \mu \mathrm{L}$ of mixture was employed to modify the electrodes. It was left to dry for approximately $45 \mathrm{~min}$.

\subsection{Electrochemical measurements}

In the case of the multiplexed electrochemical platform, to obtain analytical signals that can be correlated with the concentration of glucose, drops of measuring solution are deposited onto each paper-based electrode, one by each. This is made in such a way that the solution contacts with both, auxiliary and reference electrodes. In the case of using the automatic sampler, this was immersed in the container with the measuring solution.

Chronoamperograms were recorded applying a potential of $-0.1 \mathrm{~V}$ for $50 \mathrm{~s}$. The analytical signal is the current intensity measured at that time. A cyclic voltammogram recorded in a $1 \mathrm{mM}$ ferrocyanide solution (Figure S4) shows that at this potential there is no electrochemical oxidation of ferrocyanide. Then, the intensity obtained is only due to the reduction on the surface of the working electrode of the ferricyanide previously generated by the enzymatic reaction. Therefore, it is directly related to the concentration of glucose. The chain of enzymatic reactions can be seen in the schematics of Figure S3 (in Supplementary Data).

Each paper-based electrode and microfluidic tongue was used only once, but the connector header can be reused for several times (e.g., for performing a calibration curve and measuring food samples) without affecting the signal.

\section{Results and Discussion}


Electrochemical paper-based platforms have demonstrated their utility and so the use of carbon ink-based electrodes. Single platforms can be completed with: i) multiplexing strategies and ii) integration of a sampling step. This will advance the field of paper-based platforms towards the fully integration of the analytical process.

\subsection{Development of the multiplexed platform}

The preparation of the multiplexed platform is even easier than the single one. Once paper is wax-printed with several rows of patterned circles (Figure S1) and heated for wax diffusion, carbon ink is deposited on one side and cured. Paper is cut in rows of eight circles and, once it is modified with the enzymes and ferrocyanide, a row is clipped between the gold-plated pins of the connector. A multipotentiostat (eight channels in this case) is required to perform simultaneous measurements. Addition of glucose initiates the enzymatic cascade and produces ferricyanide that will be reduced on the surface of the carbon ink by application of the appropriate potential. The volume of the measuring solution is very important to ensure a good contact between the drop and the reference and auxiliary electrodes. Therefore, first of all, a test was performed to optimize the volume of the measuring solution. Table $S 1$ in Supplementary Data shows the results obtained when 10 and $15 \mu \mathrm{L}$ of a $5 \mathrm{mM}$ glucose solution were employed. As this table shows, both volumes gave good results. However, the reproducibility (for each node, between nodes and global) is much better for a volume of $15 \mu \mathrm{L}$. Therefore, this was the one selected to continue working with the array of biosensors.

In order to demonstrate the performance of the multiplexed platform, a calibration curve was carried out with the array of paper-based biosensors. Since they allow to perform eight measurements simultaneously, different glucose solutions were measured at once, obtaining the different points of a calibration plot at the same time. Chronoamperograms were recorded at $-0.1 \mathrm{~V}$ employing $15 \mu \mathrm{L}$ of solutions with increasing glucose concentration. As can be seen in Figure 3, a linear relationship between the current intensity measured at $50 \mathrm{~s}$ and the glucose concentration was obtained in the range comprised between 0.5 and $15 \mathrm{mM}$, according to the equation $i_{50 \mathrm{~s}}(\mu \mathrm{A})=-1.33$ [Glucose] $(\mathrm{mM})-1.33$ with a $\mathrm{R}^{2}$ of 0.998 . This calibration graph was obtained by taking measurements for the eight different concentrations in the same array of electrochemical cells, one per electrochemical cell, taking only $50 \mathrm{~s}$. This was repeated successively in five arrays. The limit of 
detection, calculated as three times the standard deviation of the intercept divided by the slope, was $0.4 \mathrm{mM}$. Since the normal range of glucose concentration in blood for healthy subjects is comprised between 4.4 and $6.6 \mathrm{mM}$ (Wang, 2008), and this is higher for diabetes patients, this concentration range and limit of detection make the device useful for the analysis of clinical samples in the diagnosis of diabetes mellitus and the monitoring of glucose levels.

In order to evaluate the reproducibility between arrays of biosensors, different calibration graphs were performed in three different days and with different solutions of buffer, enzymes, ferrocyanide and glucose. As shown in Table 1, the arrays of glucose biosensors show an excellent reproducibility with a relative standard deviation (RSD) for the slope of $1.1 \%(n=3)$. The three calibration plots are represented in Figure S5. It is important to note that each day, for each calibration, five arrays of biosensors were used, in order to have five replicates of each point of the calibration graph.

With this multiplexed platform, glucose was quantified in food samples such as orange juice and cola beverages. A very simple sample pretreatment was necessary: only in the case of the cola beverage, the sample was degassed with the help of a stirrer. After that, both samples were diluted in $0.1 \mathrm{M}$ Tris $-\mathrm{HNO}_{3}$ buffer, pH 7.0 until their concentration was within the linear range of the biosensor. The dilution made was 1:20 in both cases. The change in the $\mathrm{pH}$ after dilution was negligible in both cases. Cola beverage and orange juice changed from 2.42 and 3.90 to 6.95 and 6.50 , respectively. Then, several chronoamperograms were recorded in the same way as for the rest of the standard solutions. The results were compared to those acquired with a commercially available enzymatic kit with spectrophotometric detection, as can be seen in Table 2. The mean values of both methodologies were statistically compared through a Student's $t$ test (Miller and Miller, 2010): the $t$ values calculated for the samples were lower than the tabulated $t$ value for two degrees of freedom and a 0.05 significance level. Hence, we conclude that there were no significant differences between the values of glucose concentrations determined by the two different procedures, demonstrating the accuracy of the methodology.

The development of multiplexed platforms has many advantages, mainly that: i) standards and samples could be measured in the same device (e.g., five standards and three samples), which simplifies methodologies, ii) precision increases notably since measurements can be performed under the same environmental conditions 
(temperature, humidity...), which is especially important when bioreagents are concerned, and iii) analysis time and cost decrease, the latter due not only to the decrease in consumables but also in the analysis time.

\subsection{Design and performance of the sampling step}

Sampling is one of the steps of the analytical process that is of paramount relevance when decentralized analysis is concerned. One of the main advantages of paper (understanding paper as the cellulosic and non-cellulosic ensemble of fibers in planar disposition) is that it allows passive transport by capillarity. Including this important operation in the device, we advance towards more complete lab-on-a-chip devices (lab-on-paper). With this in mind, we have designed a microfluidic paper "tongue" that allows the collection of the sample from the container and its transport to the paper-based electrochemical cell. It is made of a glass-fiber conjugate pad and has a $T$ shape: it is $27-\mathrm{mm}$ long and $1.5-\mathrm{mm}$ wide. In the case of the inlet, the simplest design (straight cut) has been selected. The sampler is placed in such a way that the upper part of the $\mathrm{T}$ (5-mm long and 1.5-mm wide) occupies the centre of the cell. The $\mathrm{T}$ shape has many advantages since it allows: i) to deliver the sample in a more uniform way, distributing it more homogeneously, ii) to clip the sampler between the two electrodes, auxiliary and reference, in a simple manner and iii) to guarantee the ionic contact between these two electrodes. On the other hand, the second layer, the Whatman paper, is also multifunctional since: i) it receives the sample delivered by the tongue, ii) it contains the carbon ink that acts as working electrode, iii) it stores enzymes and mediator for further enzymatic reaction with glucose, iii) it transports the sample to the surface of the carbon ink, iv) it contacts the auxiliary and reference electrodes and, at the same time, v) it acts as a separator between these and the carbon ink, avoiding short-circuits and allowing ionic connection.

Regarding the dimensions of the sampler, they seem to be adequate for small containers like e.g. microcentrifuge tubes, but the sampler could be enlarged if a bottle, a longer test tube or other similar containers are employed. Regarding the material, the glass-fiber conjugate pad, commonly used in lateral flow immunoassays, has the ideal thickness to put it along with the paper-based working electrode and the gold-plated pins. It also absorbs the solution rapidly allowing the appropriate connection between the three electrodes and the sensor phase in the devices here fabricated. In addition, glass-fiber membranes do not 
show affinity towards proteins (Bahadır and Sezgintürk, 2016; Hu et al., 2014), so it would be also a good option if other analytes were used.

First, to check that the sampler works properly, it was immersed in a container with a dye (methylene blue). We observed that the solution wets completely the tongue and the electrochemical cell in approximately $30 \mathrm{~s}$ (Figure 2b), a short time, even when capillary action opposes gravity. However, if longer channels want to be employed and this effect becomes important, the sampler could be positioned in such a way that both forces act in the same direction to increase flow rate (Channon et al., 2018).

Whatman paper is wetted by direct contact with the glass-fiber conjugate pad used as sampler. Although evaporation is a phenomenon that occurs continuously, and especially here where a small volume is used, it seems not to be very relevant since measurements are taken immediately and only for $50 \mathrm{~s}$. On the other hand, the sampler could be sandwiched between pieces of tape to avoid evaporation if this issue becomes important. When using open porous devices that work under capillary action, apart from sample evaporation, inefficient sample delivery to the detection zone can be caused by sample retention by the cellulose network ( $\mathrm{Li}$ et al., 2012). Although it has been suggested for metal complexes that $50 \%$ or more of the initial sample never reached the detection zone (Nguyen et al., 2018), in our case, where glucose is implied, significant retention was not observed. On the other hand, wax barriers that, although hydrophobic, are porous and can provide potential modes for sample retention, are not required since a 1D-sampler is employed. Moreover, flow rate has decreased with the addition of wax barriers (although an increased signal for colorimetric detection schemes has been reported) (Hong and Kim, 2015). In our case, although enzymatic reactions are taken place, and usually slow flow rates are desired, here they take place at the detection area where bioreagents are immobilized. Then, this requirement does not have to be considered.

The feasibility of the microfluidic tongue was demonstrated by integrating it with the bienzymatic glucose biosensor. In this case, the paper-based electrode is prepared as before, depositing $5 \mu \mathrm{L}$ of the mixture of enzymes and ferrocyanide and letting dry. Then, the sampler is coupled to the electrochemical platform, as indicated in Figure 2. After sampling, the electrochemical measurement is performed. Due to the design and dimensions of the platform, the interaction of glucose with molecules of enzymes and the transport of ferricyanide to the electrode surface is 
extremely effective when compared with traditional biosensors (Kang et al., 2007), that employed cells of e.g. $20 \mathrm{~mL}$ and worked under stirring for efficient mass transport. Moreover, the whole analysis time, from sampling to signal recording, is only $80 \mathrm{~s}$ (30 for collecting the sample and $50 \mathrm{~s}$ for performing the chronoamperograms).

Different calibration curves have been done in different days and with different solutions in order to evaluate the feasibility and reproducibility of the system (Figure 4). A comparison between three calibration plots is shown in Table 3. Even as the microfluidic "tongue" and the electrodes are handmade, the reproducibility is very good. Moreover, the use of this sampler avoids the use of micropipettes, simplifying enormously the procedure of measuring. It can be seen the good precision of the slopes when compared with those of Table 1, corresponding to three arrays of electrochemical cells, made several months before $(-1.33 \pm 0.02 \mathrm{vs}$. $-1.34 \pm 0.02 \mu \mathrm{A} / \mathrm{mM})$, which indicates the robustness of the methodology. This could be used to simplify the methodology even more, reducing also cost and time, since the number of standards could be decreased, or even a daily calibration curve avoided. Moreover, the integration of special papers/materials that isolate plasma from blood (e.g., blood separation paper (Noiphung et al., 2013)) with the microfluidic strip could allow to perform whole blood glucose analysis.

The production of both designs, here developed, could be pilot-scaled since the materials needed are commercial or very easy to fabricate: i) mass-produced connections that consist of a rack of the required number of pins; ii) wax-patterned paper obtained with a wax-printer and e.g., 168 working areas can be obtained per paper sheet, iii) the deposition of the carbon ink and the mixture of enzymes and mediator was made with a micropipette and could be easily automatized, iv) the commercial glass-fiber strip is T-shape cut with scissors but could be easily cut using a customized patterned drill. Furthermore, the two approaches presented in this work could also be coupled, if required, reducing enormously the time of analysis. The multiplexed electrochemical platform combined with the microfluidic tongue could be an interesting strategy, to e.g., perform electrochemical measurements in an ELISA plate. Since each plate has rows of 8 wells, it fits perfectly with the paperbased platform (Figure 5). Then, the integration of a sampler and the possibility of performing simultaneous measurements sets the stage for the development of promising applications, especially in areas where decentralized and low-cost analysis is required. 


\section{Conclusions}

In this work, we have advanced in the utility of electrochemical paper-based platforms in order to allow multiplexing and sampling in biosensing. As a proof of concept, they have been tested in a biosensor for glucose determination, showing a wide linear range of concentrations and a very good reproducibility. These devices advance in the trends of miniaturization, automation, simplification, reduction of costs and analysis time, and are very promising for analytical applications in fields where decentralized analysis is usually required.

\section{Acknowledgements}

This work has been supported by the FC15-GRUPIN-021 project from the Asturias Regional Government and CTQ2014-58826-R project from the Spanish Ministry of Economy and Competitiveness (MINECO).

\section{Supplementary material}

Supplementary data associated with this article can be found in the online version.

\section{References}

Ainla, A., Mousavi, M.P.S., Tsaloglou, M.-N., Redston, J., Bell, J.G., Fernández-Abedul, M.T., Whitesides, G.M., 2018. Open-Source Potentiostat for Wireless Electrochemical Detection with Smartphones. Anal. Chem. 90, 6240-6246. https://doi.org/10.1021/acs.analchem.8b00850

Amor-Gutiérrez, O., Costa Rama, E., Costa-García, A., Fernández-Abedul, M.T., 2017. Paper-based maskless enzymatic sensor for glucose determination combining ink and wire electrodes. Biosens. Bioelectron. 93, 40-45. https://doi.org/10.1016/j.bios.2016.11.008

Arduini, F., Cinti, S., Scognamiglio, V., Moscone, D., Palleschi, G., 2017. How cuttingedge technologies impact the design of electrochemical (bio)sensors for 
environmental analysis. A review. Anal. Chim. Acta 959, 15-42.

https://doi.org/10.1016/j.aca.2016.12.035

Bahadır, E.B., Sezgintürk, M.K., 2016. Lateral flow assays: Principles, designs and labels. TrAC - Trends Anal. Chem. 82, 286-306. https://doi.org/10.1016/j.trac.2016.06.006

Bernalte, E., Foster, C.W., Brownson, D.A.C., Mosna, M., Smith, G.C., Banks, C.E., 2016. Pencil it in: Exploring the feasibility of hand-drawn pencil electrochemical sensors and their direct comparison to screen-printed electrodes. Biosensors 6, 45. https://doi.org/10.3390/bios6030045

Biscay, J., Rama, E.C., García, M.B.G., Carrazón, J.M.P., García, A.C., 2011. Enzymatic sensor using mediator-screen-printed carbon electrodes. Electroanalysis 23, 209214.

Cate, D.M., Adkins, J.A., Mettakoonpitak, J., Henry, C.S., 2015. Recent Developments in Paper-Based Microfluidic Devices. Anal. Chem. 87, 19-41. https://doi.org/10.1021/ac503968p

Channon, R.B., Nguyen, M.P., Scorzelli, A.G., Henry, E.M., Volckens, J., Dandy, D.S., Henry, C.S., 2018. Rapid flow in multilayer microfluidic paper-based analytical devices. Lab Chip 18, 793-802. https://doi.org/10.1039/c7lc01300k

Cinti, S., Basso, M., Moscone, D., Arduini, F., 2017a. A paper-based nanomodified electrochemical biosensor for ethanol detection in beers. Anal. Chim. Acta 960, 123130. https://doi.org/10.1016/j.aca.2017.01.010

Cinti, S., Minotti, C., Moscone, D., Palleschi, G., Arduini, F., 2017b. Fully integrated readyto-use paper-based electrochemical biosensor to detect nerve agents. Biosens. Bioelectron. 93, 46-51. https://doi.org/10.1016/j.bios.2016.10.091

Cunningham, J.C., DeGregory, P.R., Crooks, R.M., 2016. New Functionalities for PaperBased Sensors Lead to Simplified User Operation, Lower Limits of Detection, and New Applications. Annu. Rev. Anal. Chem. 9, 4.1-4.20. https://doi.org/10.1146/annurev-anchem-071015-041605

Dossi, N., Toniolo, R., Impellizzieri, F., Bontempelli, G., 2014. Doped pencil leads for drawing modified electrodes on paper-based electrochemical devices. J. Electroanal. Chem. 722-723, 90-94. 
Galant, A.L., Kaufman, R.C., Wilson, J.D., 2015. Glucose: Detection and Analysis. Food Chem. 188, 149-160. https://doi.org/10.1016/j.foodchem.2015.04.071

Gałuszka, A., Migaszewski, Z., Namieśnik, J., 2013. The 12 principles of green analytical chemistry and the SIGNIFICANCE mnemonic of green analytical practices. TrAC Trends Anal. Chem. 50, 78-84. https://doi.org/10.1016/j.trac.2013.04.010

Glavan, A.C., Christodouleas, D.C., Mosadegh, B., Yu, H.D., Smith, B.S., Lessing, J., Fernández-Abedul, M.T., Whitesides, G.M., 2014. Folding analytical devices for electrochemical ELISA in hydrophobic RHpaper. Anal. Chem. 86, 11999-12007. https://doi.org/10.1021/ac5020782

Hamedi, M.M., Ainla, A., Güder, F., Christodouleas, D.C., Fernández-Abedul, M.T., Whitesides, G.M., 2016. Integrating Electronics and Microfluidics on Paper. Adv. Mater. 28, 5054-5063. https://doi.org/10.1002/adma.201505823

Hong, S., Kim, W., 2015. Dynamics of water imbibition through paper channels with wax boundaries. Microfluid. Nanofluidics 19, 845-853. https://doi.org/10.1007/s10404015-1611-3

Hu, J., Wang, S.Q., Wang, L., Li, F., Pingguan-Murphy, B., Lu, T.J., Xu, F., 2014. Advances in paper-based point-of-care diagnostics. Biosens. Bioelectron. 54, 585597. https://doi.org/10.1016/j.bios.2013.10.075

Kang, X., Mai, Z., Zou, X., Cai, P., Mo, J., 2007. A novel glucose biosensor based on immobilization of glucose oxidase in chitosan on a glassy carbon electrode modified with gold-platinum alloy nanoparticles/multiwall carbon nanotubes. Anal. Biochem. 369, 71-79. https://doi.org/10.1016/j.ab.2007.07.005

Li, X., Ballerini, D.R., Shen, W., 2012. A perspective on paper-based microfluidics: Current status and future trends. Biomicrofluidics 6, 011301-111313. https://doi.org/10.1063/1.3687398

Liao, Z., Wang, J., Zhang, P., Zhang, Y., Miao, Y., Gao, S., Deng, Y., Geng, L., 2018. Recent advances in microfluidic chip integrated electronic biosensors for multiplexed detection. Biosens. Bioelectron. 121, 272-280. https://doi.org/10.1016/j.bios.2018.08.061

Martín-Yerga, D., González-García, M.B., Costa-García, A., 2013. Biosensor array based on the in situ detection of quantum dots as electrochemical label. Sensors Actuators 
B Chem. 182, 184-189. https://doi.org/10.1016/j.talanta.2014.07.010

Martinez, A.W., Phillips, S.T., Butte, M.J., Whitesides, G.M., 2007. Patterned paper as a platform for inexpensive, low-volume, portable bioassays. Angew. Chemie - Int. Ed. 46, 1318-1320. https://doi.org/10.1002/anie.200603817

Martinez, A.W., Phillips, S.T., Whitesides, G.M., Carrilho, E., 2010. Diagnostics for the developing world: Microfluidic paper-based analytical devices. Anal. Chem. 82, 3-10. https://doi.org/10.1021/ac9013989

Mazzu-Nascimento, T., Donofrio, F.C., Bianchi, B.C., Travensolo, R. de F., Junior, J.B.S., Moraes, D.A., Morbioli, G.G., Segato, T.P., Varanda, L.C., Carrilho, E., 2017. Paperbased microfluidics immunoassay for detection of canine distemper virus. Brazilian Arch. Biol. Technol. 60, 1-11. https://doi.org/10.1590/1678-4324-2016160317

Mettakoonpitak, J., Boehle, K., Nantaphol, S., Teengam, P., Adkins, J.A., Srisa-Art, M., Henry, C.S., 2016. Electrochemistry on Paper-based Analytical Devices: A Review. Electroanalysis 28, 1420-1436. https://doi.org/10.1002/elan.201501143

Miller, J., Miller, J.C., 2010. Statistics and Chemometrics for Analytical Chemistry, 6th ed. ed. Prentice Hall, Harlow, U.K.

Nanni, P.I., González-López, A., Núnez-Bajo, E., Madrid, R., Fernandez Abedul, M.T., 2018. Staple-based paper electrochemical platform for celiac disease diagnosis. ChemElectroChem 5, 4036-4045. https://doi.org/10.1002/celc.201800743

Nemiroski, A., Christodouleas, D.C., Hennek, J.W., Kumar, A.A., Maxwell, E.J., Fernández-Abedul, M.T., Whitesides, G.M., 2014. Universal mobile electrochemical detector designed for use in resource-limited applications. Proc. Natl. Acad. Sci. U. S. A. 111, 11984-11989. https://doi.org/10.1073/pnas.1405679111

Nguyen, M.P., Meredith, N.A., Kelly, S.P., Henry, C.S., 2018. Design considerations for reducing sample loss in microfluidic paper-based analytical devices. Anal. Chim. Acta 1017, 20-25. https://doi.org/10.1016/j.aca.2018.01.036

Nie, Z., Nijhuis, C.A., Gong, J., Chen, X., Kumachev, A., Martinez, A.W., Narovlyansky, M., Whitesides, G.M., 2010. Electrochemical sensing in paper-based microfluidic devices. Lab Chip 10, 477-483. https://doi.org/10.1039/B917150A

Noiphung, J., Songjaroen, T., Dungchai, W., Henry, C.S., Chailapakul, O., 
Laiwattanapaisal, W., 2013. Electrochemical detection of glucose from whole blood using paper-based microfluidic devices. Anal. Chim. Acta 788, 39-45.

https://doi.org/10.1016/j.aca.2013.06.021

Rama, E.C., Costa-García, A., Fernández-Abedul, M.T., 2017. Pin-based electrochemical glucose sensor with multiplexing possibilities. Biosens. Bioelectron. 88, 34-40. https://doi.org/10.1016/j.bios.2016.06.068

Rodríguez, M.O., Covián, L.B., García, A.C., Blanco-López, M.C., 2016. Silver and gold enhancement methods for lateral flow immunoassays. Talanta 148, 272-278. https://doi.org/10.1016/j.talanta.2015.10.068

Russo, A., Ahn, B.Y., Adams, J.J., Duoss, E.B., Bernhard, J.T., Lewis, J.A., 2011. Pen-onpaper flexible electronics. Adv. Mater. 23, 3426-3430.

Song, J., Liu, C., Mauk, M.G., Peng, J., Schoenfeld, T., Bau, H.H., 2018. A Multifunctional Reactor with Dry-Stored Reagents for Enzymatic Amplification of Nucleic Acids. Anal. Chem. 90, 1209-1216. https://doi.org/10.1021/acs.analchem.7b03834

Teengam, P., Siangproh, W., Tuantranont, A., Henry, C.S., Vilaivan, T., Chailapakul, O., 2017. Electrochemical paper-based peptide nucleic acid biosensor for detecting human papillomavirus. Anal. Chim. Acta 952, 32-40. https://doi.org/10.1016/j.aca.2016.11.071

Tobjörk, D., Österbacka, R., 2011. Paper Electronics. Adv. Mater. 23, 1935-1961. https://doi.org/10.1002/adma.201004692

Wang, J., 2008. Electrochemical glucose biosensors. Chem. Rev. 108, 814-825. https://doi.org/10.1016/B978-012373738-0.50005-2

Xu, D., Huang, X., Guo, J., Ma, X., 2018. Automatic smartphone-based microfluidic biosensor system at the point of care. Biosens. Bioelectron. 110, 78-88. https://doi.org/10.1016/j.bios.2018.03.018

Yager, P., Edwards, T., Fu, E., Helton, K., Nelson, K., Tam, M.R., Weigl, B.H., 2006. Microfluidic diagnostic technologies for global public health. Nature 442, 412-418. https://doi.org/10.1038/nature05064

Yamada, K., Henares, T.G., Suzuki, K., Citterio, D., 2015. Paper-based inkjet-printed microfluidic analytical devices. Angew. Chemie - Int. Ed. 54, 5294-5310. 
https://doi.org/10.1002/anie.201411508

Yang, Y., Noviana, E., Nguyen, M.P., Geiss, B.J., Dandy, D.S., Henry, C.S., 2017. PaperBased Microfluidic Devices: Emerging Themes and Applications. Anal. Chem. 89, 71-91. https://doi.org/10.1021/acs.analchem.6b04581

Zhao, C., Thuo, M.M., Liu, X., 2013. A microfluidic paper-based electrochemical biosensor array for multiplexed detection of metabolic biomarkers. Sci. Technol. Adv. Mater. 14, 1-7. https://doi.org/10.1088/1468-6996/16/4/049501 


\section{Caption of Figures}

Figure 1. Construction of the paper-based array of electrodes: a) pictures with views (top and bottom) of the array clipped between gold-plated connectors, b) picture of an array of electrodes connected to the multipotentiostat by a commercial interface and ready to measure in $15 \mu \mathrm{L}$-drops of glucose solution, $\mathrm{c}$ ) screen capture of the Dropview 84002.0 software with eight measurements (chronoamperograms) recorded in six glucose solutions of different concentration and two samples (orange juice and cola beverage) and $d$ ) schematics of the platform including the reagents that are adsorbed.

Figure 2. Picture of: a) the different components of the microfluidic device: paperbased electrode (bottom and top views), microfluidic "tongue" and connector and b) the assembled device with indication of the time needed by the solution (in this case a methylene blue solution) to reach the electrochemical platform through the sampler. The different times depicted are 10, 20 and $30 \mathrm{~s}$.

Figure 3. Chronoamperograms recorded for glucose solutions of increasing concentrations on arrays of paper-based glucose biosensors combined with goldplated connector headers. Inset: Calibration plot using 5 different arrays of biosensors. Detection potential: $-0.1 \mathrm{~V}$ vs. a gold-plated pseudoreference pin. Analytical signal measured at $50 \mathrm{~s}$. Data given as average $\pm \operatorname{SD}(n=5)$.

Figure 4. Calibration plots made with the paper-based electrochemical platform combined with the microfluidic tongue in three different days and with different solutions. Detection potential: - $0.1 \mathrm{~V}$ vs. a gold-plated pseudoreference electrode. Analytical signal measured at $50 \mathrm{~s}$. Data given as average $\pm \operatorname{SD}(n=5)$.

Figure 5. Picture of the multiplexed platform with the 8 samplers introduced in the wells of a row of an ELISA microtiter plate. 


\section{Tables}

Table 1. Reproducibility study with data obtained using three different arrays of biosensors to carry out three calibration graphs in different days and with different solutions.

\begin{tabular}{|c|c|c|c|}
\hline $\begin{array}{c}\text { Calibration } \\
\text { plot }\end{array}$ & $\begin{array}{c}\text { Slope } \\
(\boldsymbol{\mu A} / \mathbf{m M})\end{array}$ & $\mathbf{R}^{2}$ & $\begin{array}{c}\text { Linear range } \\
(\mathbf{m M})\end{array}$ \\
\hline Day 1 & -1.33 & 0.998 & $0.5-15$ \\
\hline Day 2 & -1.35 & 0.996 & $0.5-15$ \\
\hline Day 3 & -1.32 & 0.994 & $0.5-15$ \\
\hline \hline Sensitivity & $-\mathbf{1 . 3 3 \pm 0 . 0 2}$ & \multicolumn{2}{|l}{} \\
\hline \hline $\begin{array}{c}\text { RSD } \\
\text { sensitivity }\end{array}$ & $\mathbf{1 . 1 \%}$ & \multicolumn{2}{|l}{} \\
\cline { 1 - 2 } & \multicolumn{2}{|l}{}
\end{tabular}

Table 2. Results for real food sample analysis performed with the array of biosensors. Data are given as average $\pm S D(n=5$ for the paper-based array of biosensors and $n=3$ for the spectrophotometric assay).

\begin{tabular}{|c|c|c|}
\hline Sample & $\begin{array}{c}\text { Paper-based array } \\
\text { of biosensors }\end{array}$ & $\begin{array}{c}\text { Spectrophotometric } \\
\text { assay }\end{array}$ \\
\hline $\begin{array}{c}\text { Commercial } \\
\text { orange juice }\end{array}$ & $2.72 \pm 0.2 \mathrm{~g} / 100 \mathrm{~mL}$ & $2.71 \pm 0.04 \mathrm{~g} / 100 \mathrm{~mL}$ \\
\hline $\begin{array}{c}\text { Cola } \\
\text { beverage }\end{array}$ & $2.73 \pm 0.3 \mathrm{~g} / 100 \mathrm{~mL}$ & $2.81 \pm 0.03 \mathrm{~g} / 100 \mathrm{~mL}$ \\
\hline
\end{tabular}

Table 3. Reproducibility study with data obtained from three calibration graphs carried out with the single paper-based electrochemical platform combined with the microfluidic tongue in different days and with different solutions.

\begin{tabular}{|c|c|c|c|}
\hline $\begin{array}{c}\text { Calibration } \\
\text { plot }\end{array}$ & $\begin{array}{c}\text { Slope } \\
(\boldsymbol{\mu A} / \mathbf{m M})\end{array}$ & $\mathbf{R}^{\mathbf{2}}$ & $\begin{array}{c}\text { Linear } \\
\text { range } \\
(\mathbf{m M})\end{array}$ \\
\hline Day 1 & -1.34 & 0.997 & $0.5-15$ \\
\hline Day 2 & -1.33 & 0.998 & $0.5-15$ \\
\hline Day 3 & -1.35 & 0.996 & $0.5-15$ \\
\hline \hline Sensitivity & $-\mathbf{1 . 3 4 \pm \mathbf { 0 . 0 2 }}$ & \multicolumn{2}{|c}{} \\
\cline { 1 - 2 } $\begin{array}{c}\text { RSD } \\
\text { sensitivity }\end{array}$ & $\mathbf{1 . 0} \%$ & \multicolumn{2}{|l}{} \\
\cline { 1 - 2 } & \multicolumn{2}{|l}{}
\end{tabular}


Figure 1

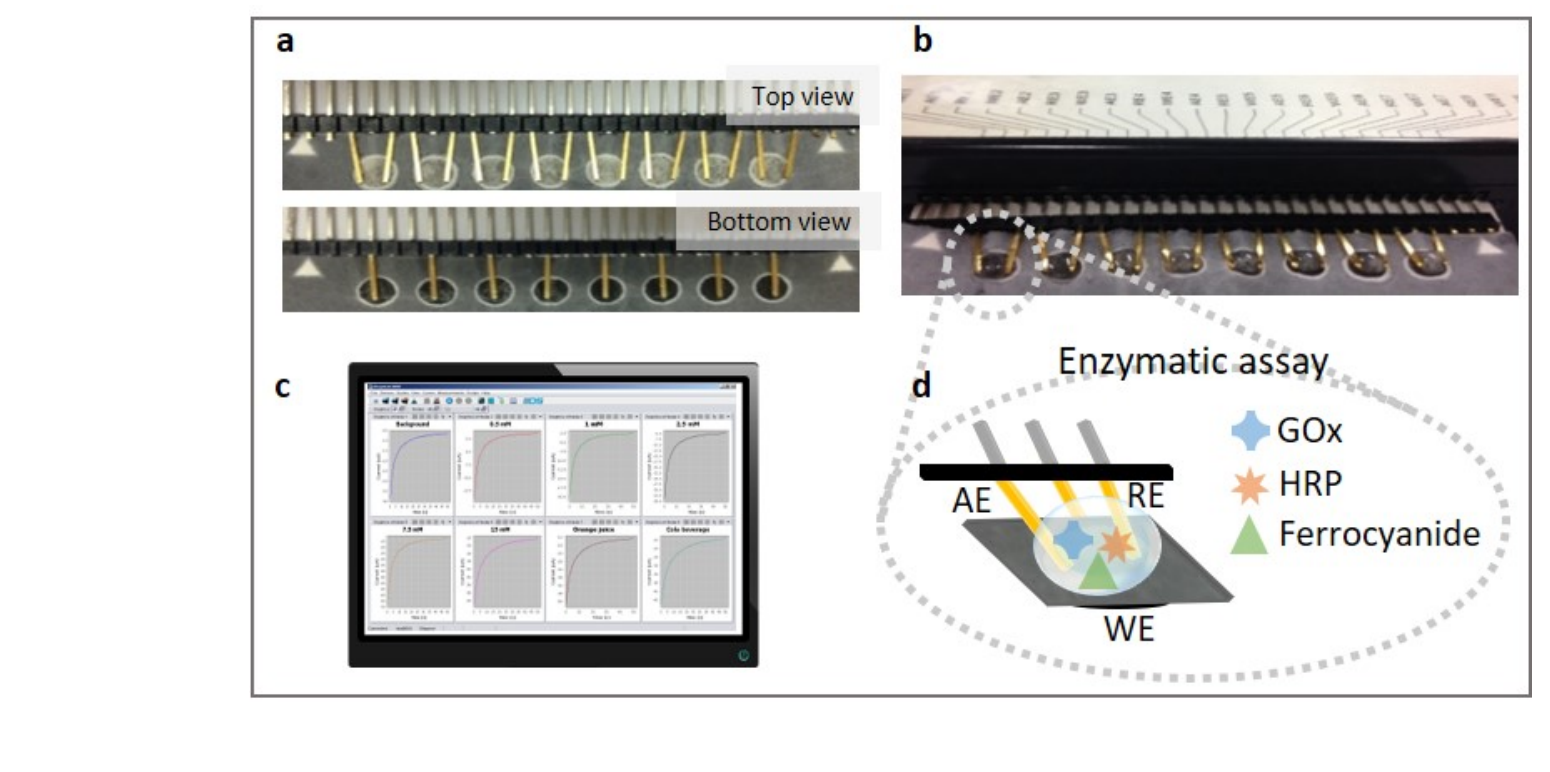

\section{Figure 1}

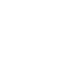

(1)
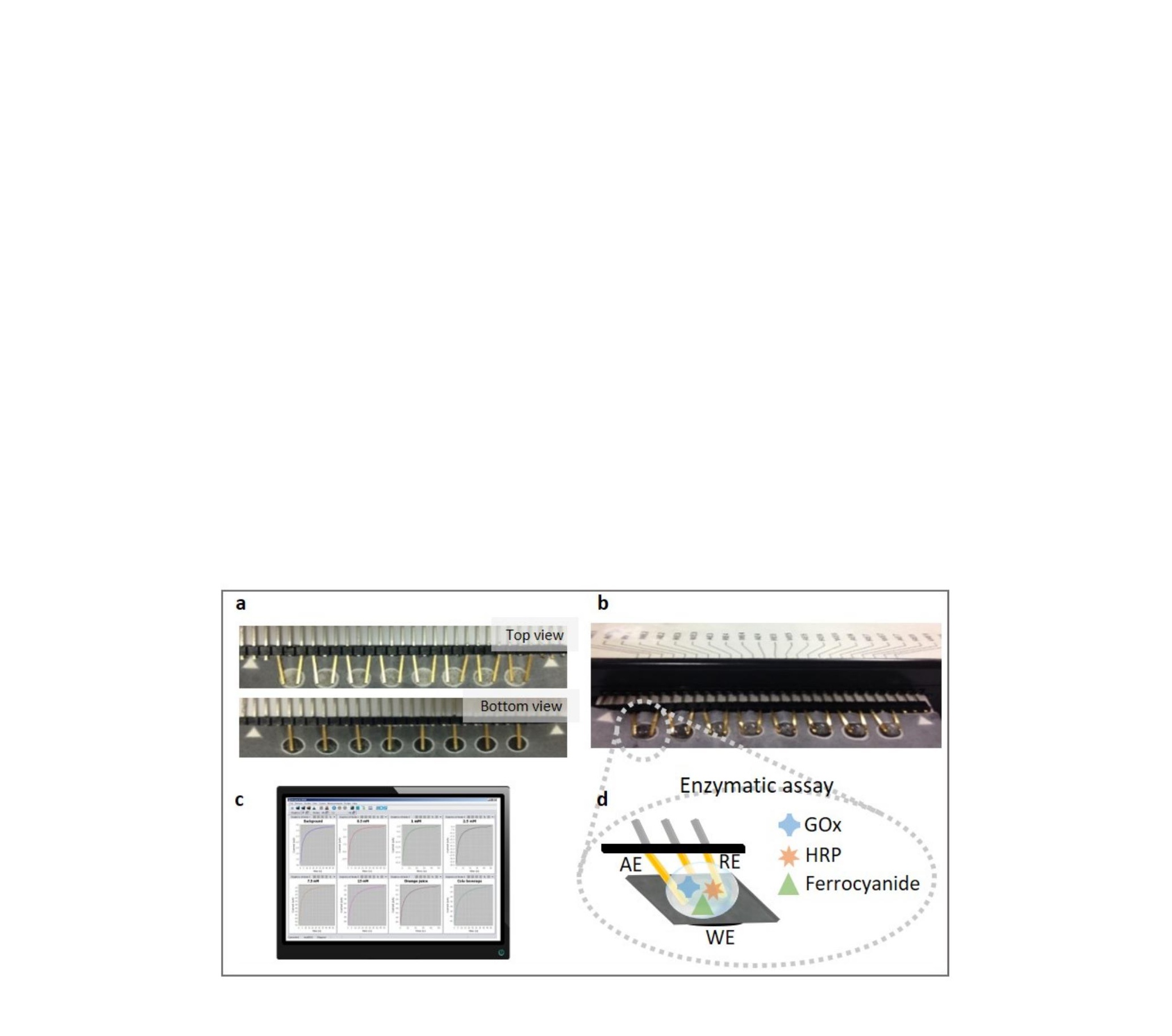


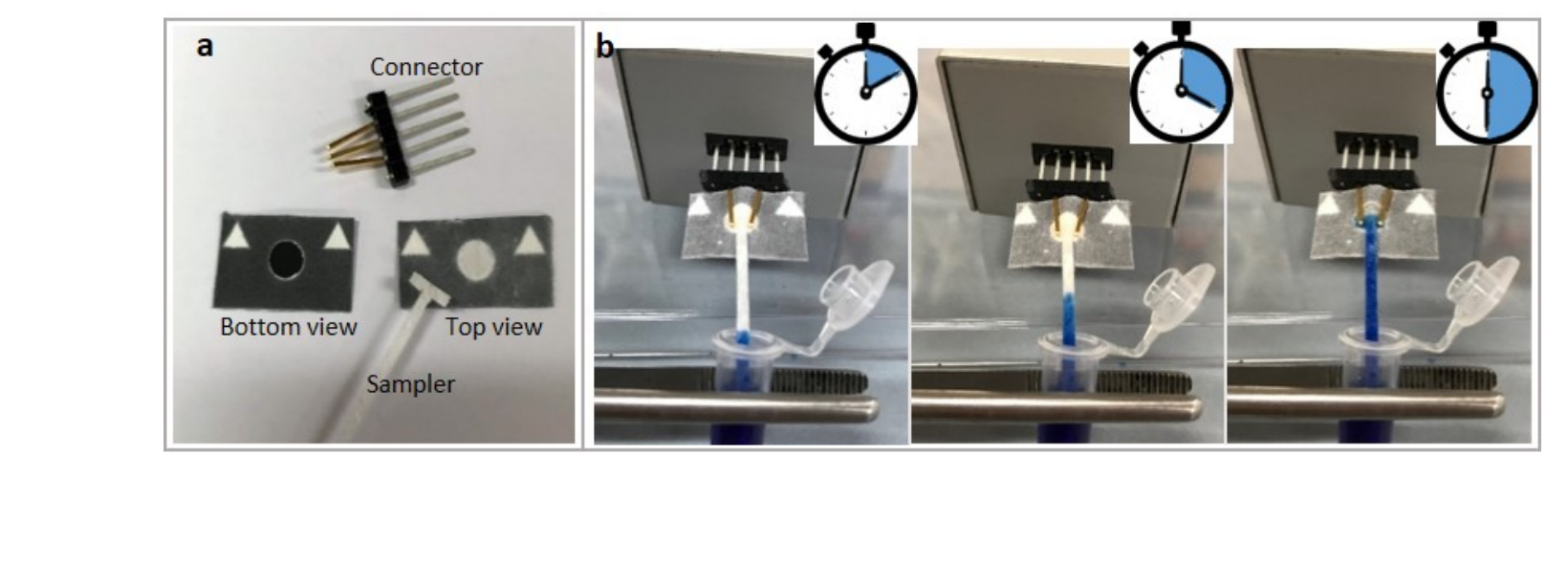

Figure 2

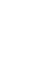

\section{Figure 2}

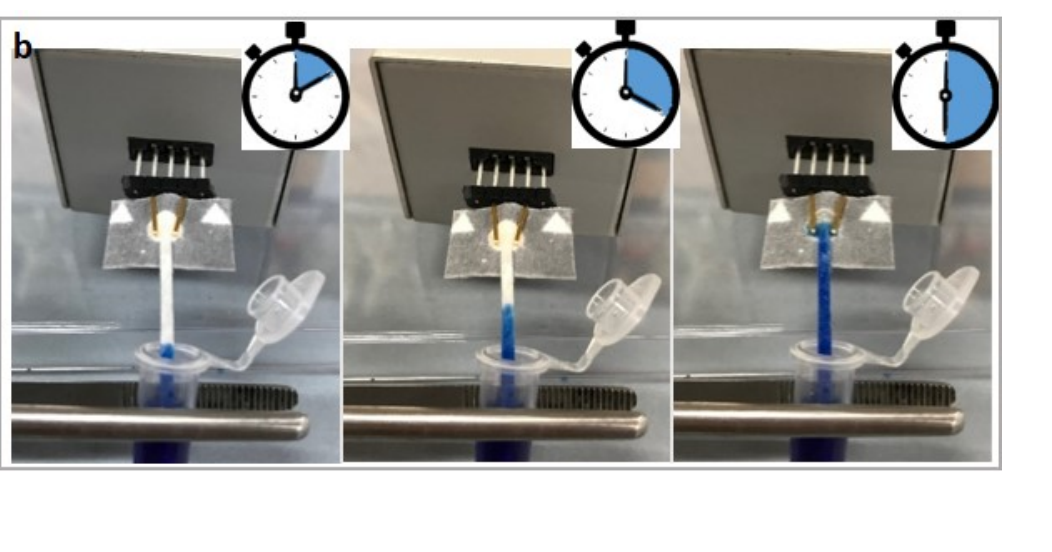

2

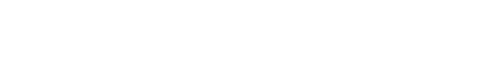
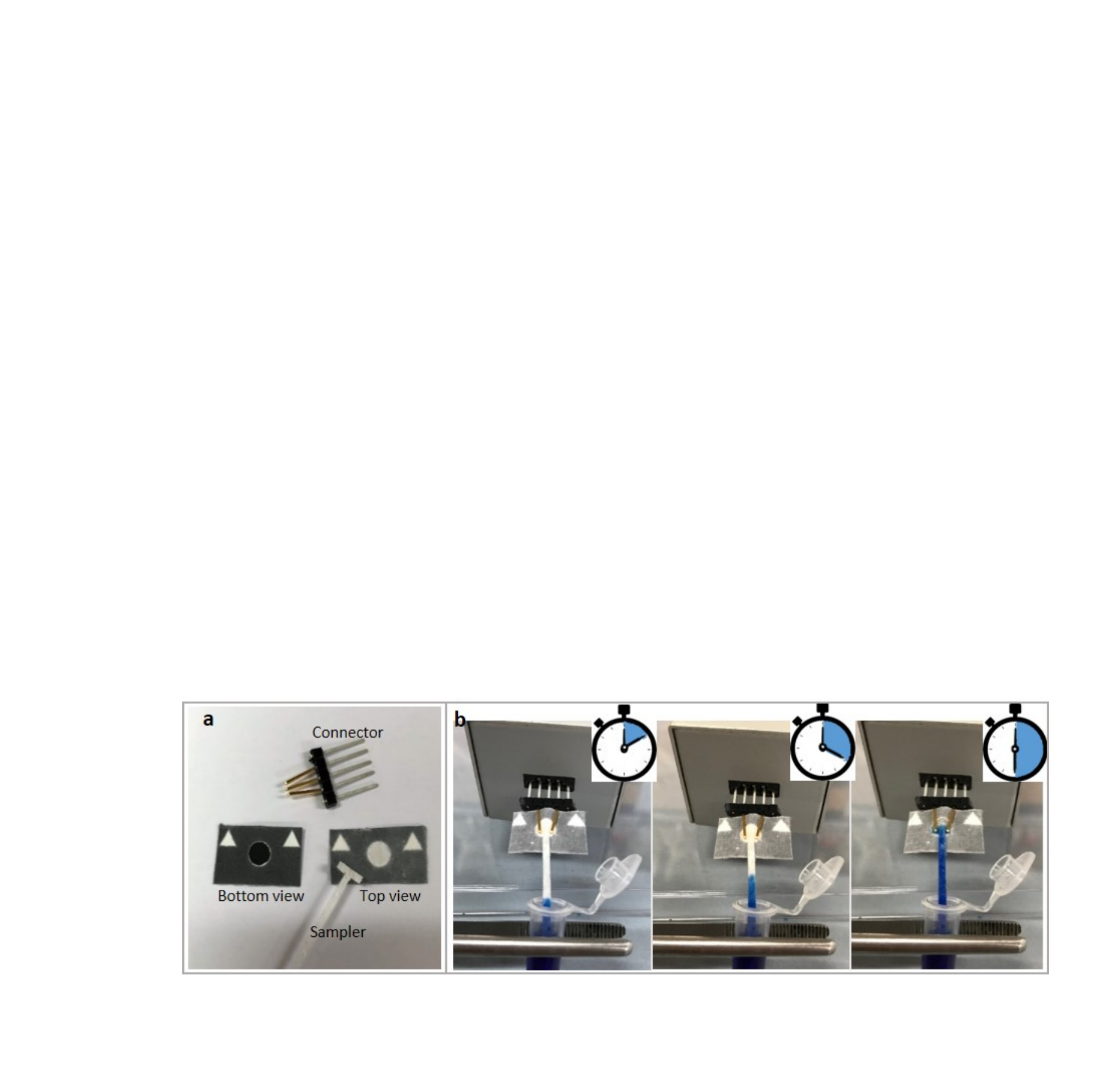

,
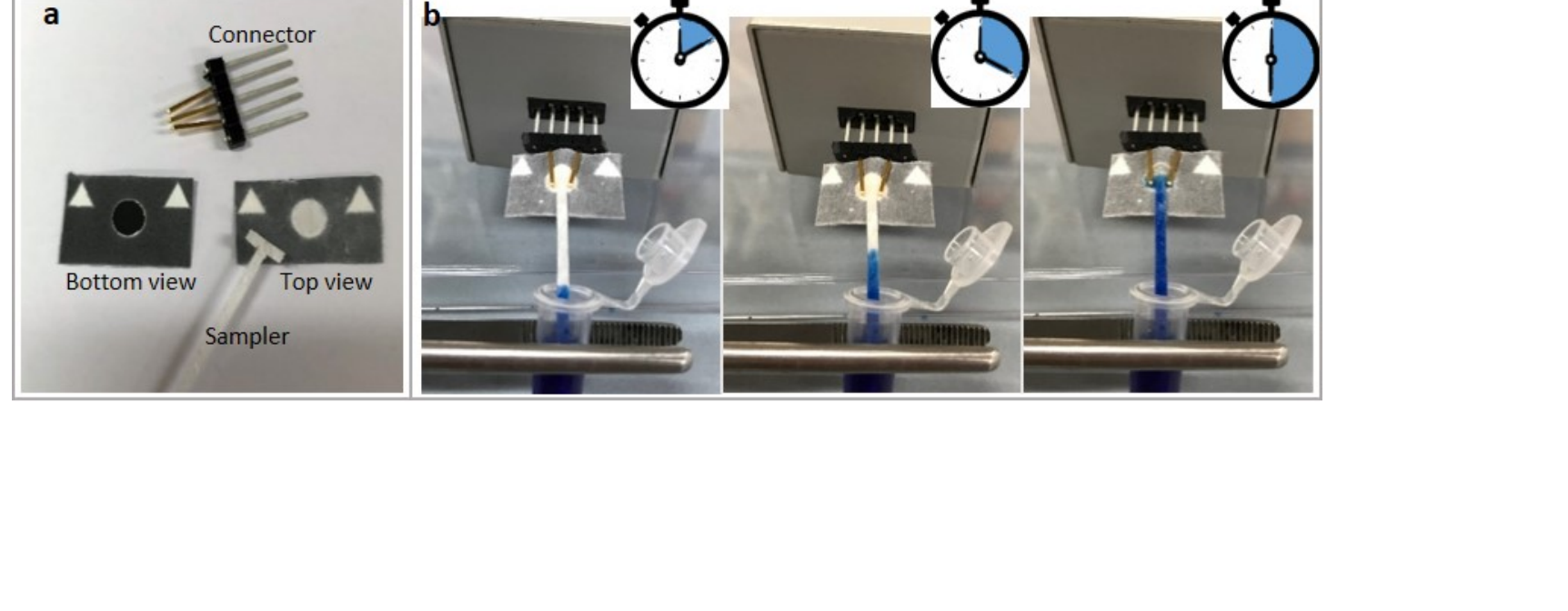
Figure(s)
Click here to download high resolution image

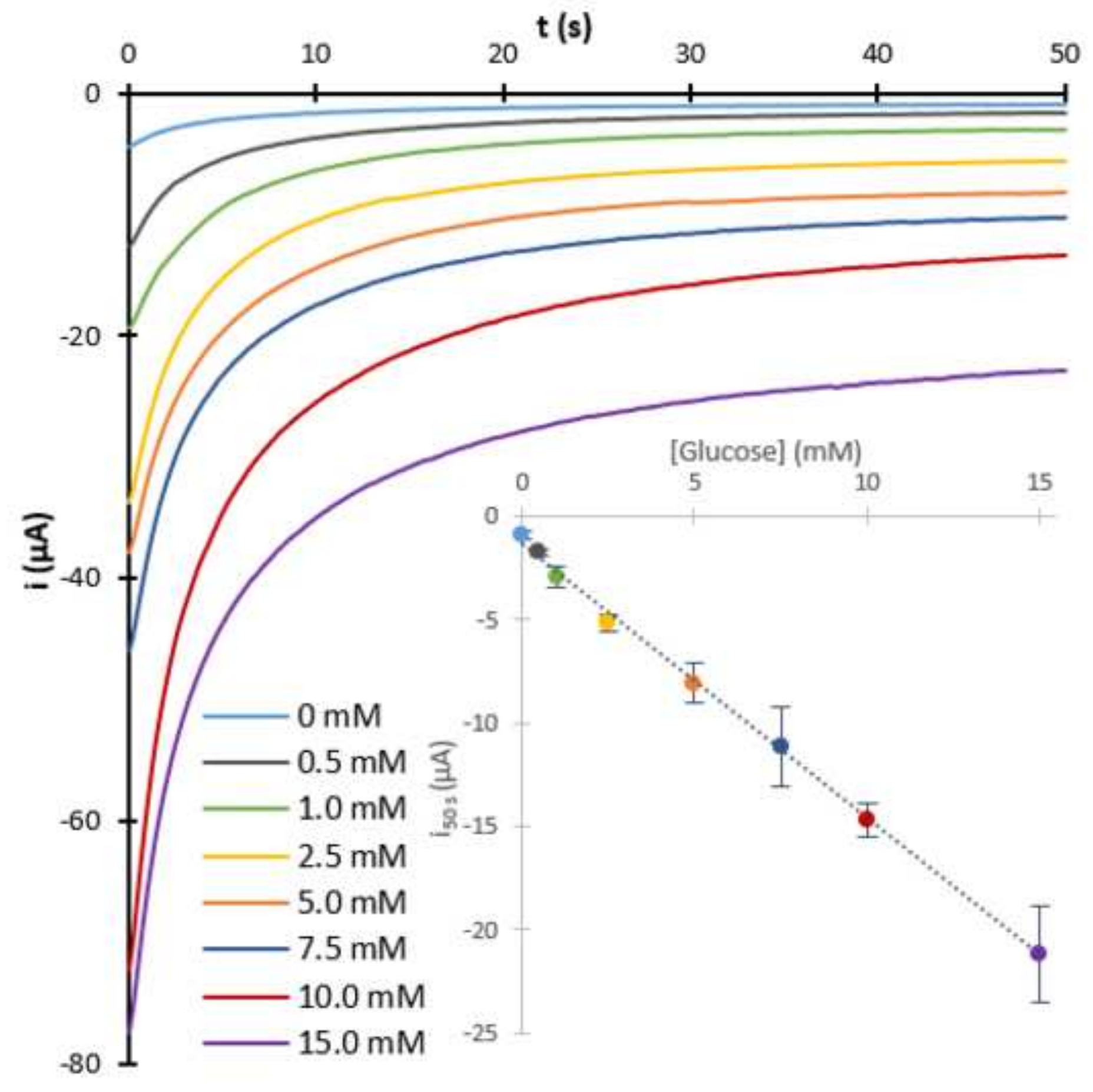




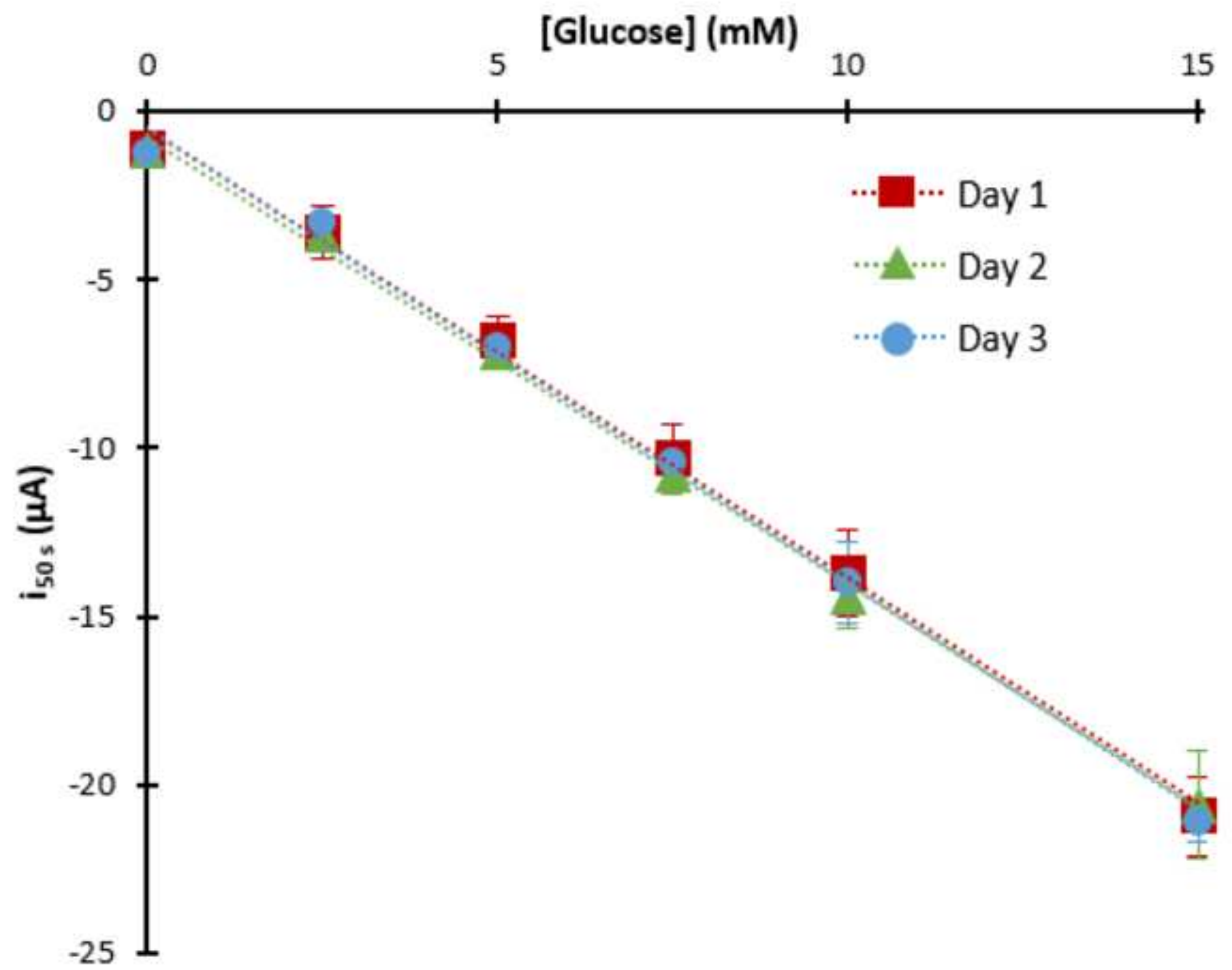


Click here to download high resolution image

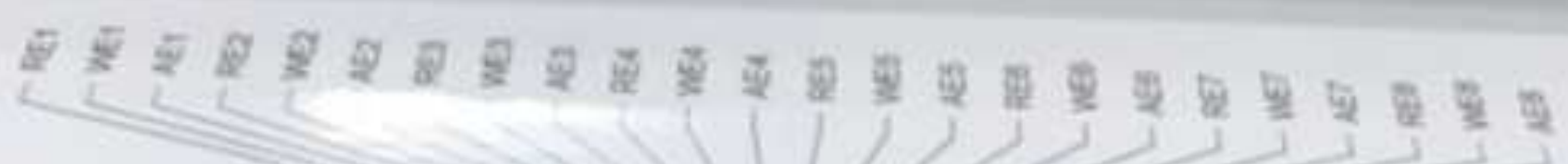

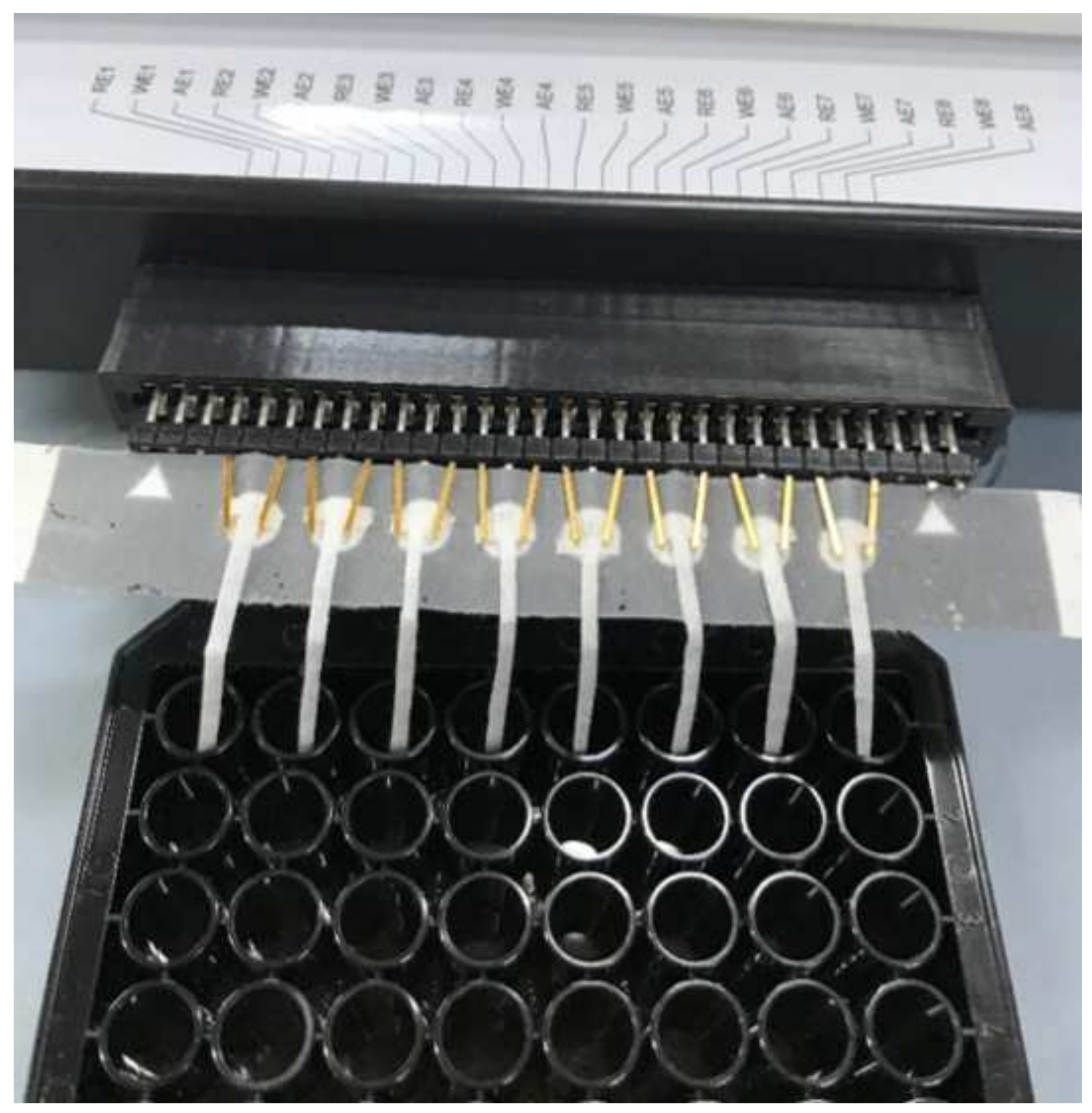


Supplementary Material
Click here to download Supplementary Material: 2-SI highlighted.docx

Supplementary Material
Click here to download Supplementary Material: 2-SI highlighted.docx

.


Departamentu de Química Física y Analítica

Department of Physical and Analytical Chemistry

\section{AUTHORSHIP STATEMENT:}

Title: Sampling and multiplexing in lab-on-paper bioelectroanalytical paper-based devices for glucose determination

Ms. Ref. No.: BIOS-D-19-00449

Authors: O. Amor-Gutiérrez, E. Costa-Rama, M. T. Fernández-Abedul

Institution: Universidad de Oviedo (Spain)

All persons who meet authorship criteria are listed as authors, and all authors certify that they have participated sufficiently in the work to take public responsibility for the content, including participation in the concept, design, analysis, writing, or revision of the manuscript. Furthermore, each author certifies that this material or similar material has not been and will not be submitted to or published in any other publication before its appearance in Biosensors and Bioelectronics.

\section{Authorship contribution}

\section{Category 1}

Conception and design of study: M.Teresa Fernández Abedul, acquisition of data: Olaya Amor Gutiérrez, analysis and/or interpretation of data: M. Teresa Fernández Abedul, Estefanía Costa Rama, Olaya Amor Gutiérrez

\section{Category 2}

Drafting the manuscript: Olaya Amor Gutiérrez, Estefanía Costa Rama

revising the manuscript critically for important intellectual content: M.Teresa Fernández Abedul,

\section{Category 3}

Approval of the version of the manuscript to be published (the names of all authors must be listed): Olaya Amor Gutierrez, Estefanía Costa Rama, M.Teresa Fernández Abedul

Acknowledgements 
Departamentu de Química Física y Analítica

Department of Physical and Analytical Chemistry

All persons who have made substantial contributions to the work reported in the manuscript (e.g., technical help, writing and editing assistance, general support), but who do not meet the criteria for authorship, are named in the Acknowledgements and have given us their written permission to be named. If we have not included an Acknowledgements, then that indicates that we have not received substantial contributions from non-authors.

This statement is signed by all the authors,

Author's name (typed)

Olaya Amor Gutiérrez

Estefanía Costa Rama

M. Teresa Fernández Abedul

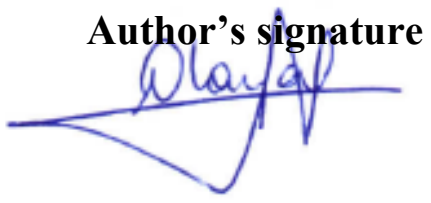

Date

2019, March 25th

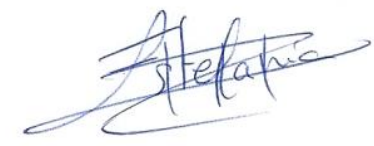

2019, March 25th

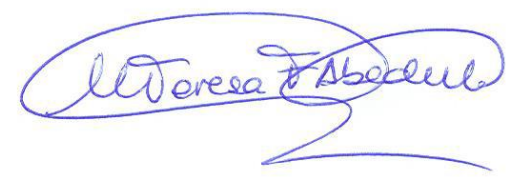

2019, March 25th 